\title{
Supplementary Material For: \\ Machine Learning Assisted Design of Highly Active Peptides for Drug Discovery
}

Sébastien Giguère ${ }^{1} *$, François Laviolette ${ }^{1}$, Mario Marchand $^{1}$, Denise Tremblay $^{2}$, Sylvain Moineau ${ }^{2}$, Éric Biron $^{3}$, Xinxia Liang ${ }^{3}$ and Jacques Corbeil ${ }^{4}$

1 Department of Computer Science and Software Engineering, Université Laval, Québec, Canada

2 Department of Biochemistry, Microbiology and Bioinformatics, Université Laval, Québec, Canada

3 Faculty of Pharmacy, Université Laval, Québec, Canada

4 Department of Molecular Medicine, Université Laval, Québec, Canada

* E-mail: Corresponding sebastien.giguere.8@ulaval.ca

\section{Expected outcome of a library given a protocol}

The proposed approach makes use of the graph $G^{h_{\mathbf{y}}}$, the protocol $P$, and a dynamic programming algorithm that exploits recurrences in the factorization of first and second order polynomials. This allows for the efficient computation of $\tau$ and $\beta$, respectively the first and second moment of $h_{\mathbf{y}}$ when peptides are drawn according to the distribution $P$ :

$$
\begin{aligned}
& \tau \stackrel{\text { def }}{=} \\
& \beta \stackrel{\text { def }}{=} \sum_{\mathbf{x} \in \mathcal{A}^{l}} P(\mathbf{x}) \cdot h_{\mathbf{y}}(\mathbf{x}) \\
& \sum_{\mathbf{x} \in \mathcal{A}^{l}} P(\mathbf{x}) \cdot h_{\mathbf{y}}(\mathbf{x})^{2} .
\end{aligned}
$$

Thus, the average and variance predicted bioactivity of peptides synthesized by the protocol are then respectively given by $\tau$ and $\beta-\tau^{2}$.

To compute these quantities efficiently, the dynamic programming algorithm, given in Algorithm 1, uses the following recurrence relations:

$$
\sum_{i=1}^{n} x_{i}=x_{n}+\sum_{i=1}^{n-1} x_{i}
$$

and

$$
\left(\sum_{i=1}^{n} x_{i}\right)^{2}=\left(\sum_{i=1}^{n-1} x_{i}\right)^{2}+2 x_{n}\left(\sum_{i=1}^{n-1} x_{i}\right)+x_{n}^{2} .
$$

Moreover, each node of the graph $G^{h_{\mathbf{y}}}$ has the following additional variables.

- $\tau[s, i]$ for the expected length of paths from the source node $\lambda$ to the node $(s, i)$.

- $\beta[s, i]$ for the expected squared length of paths from the source node $\lambda$ to the node $(s, i)$.

- $\boldsymbol{\rho}[s, i]$ is the probability of having the $k$-mers $s$ at position $i$.

After the execution of Algorithm 1, the values of $\tau$ and $\beta$ are respectively given by $\boldsymbol{\tau}[t]$ and $\beta[t]$ for the sink node $t$. 


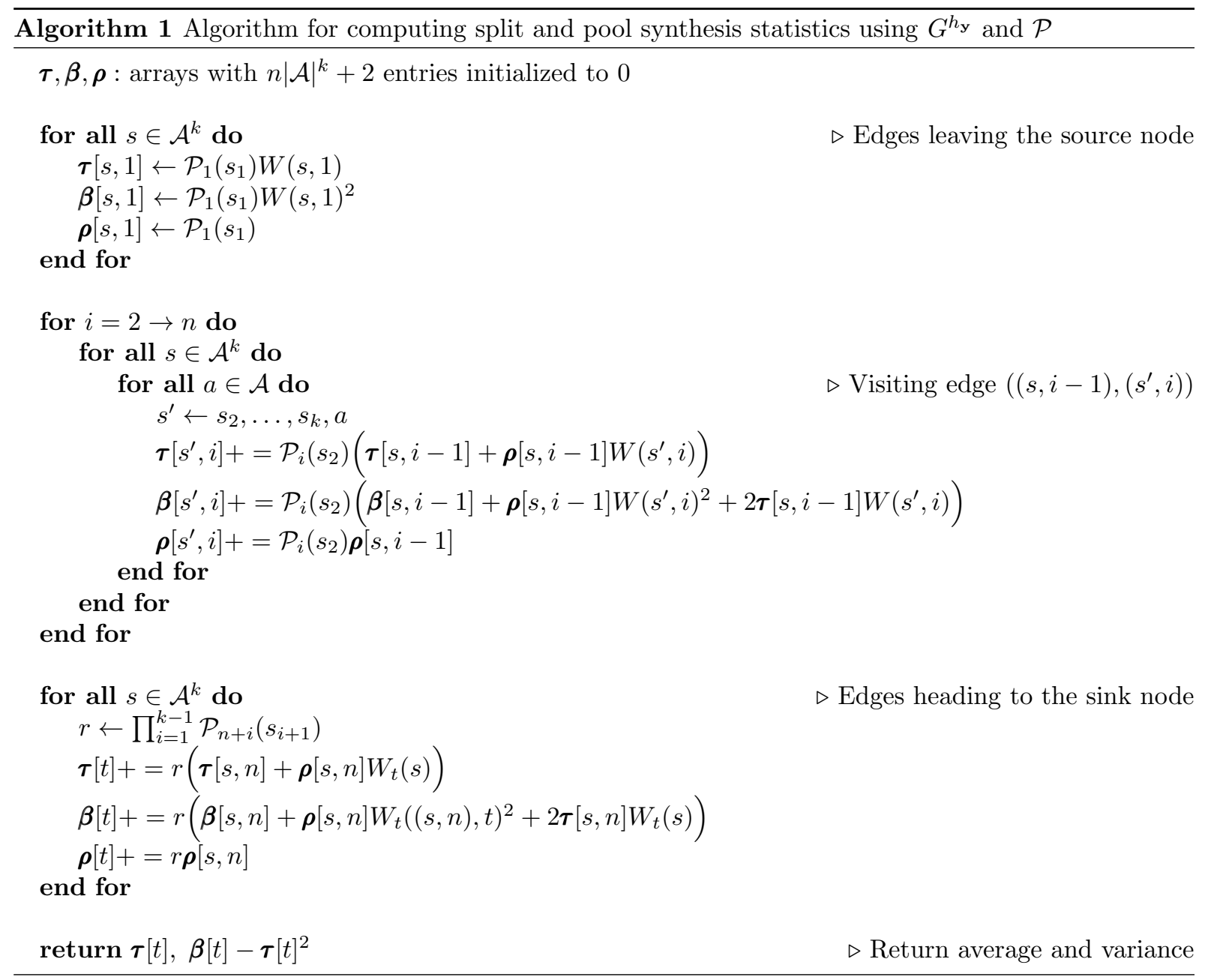




\section{Peptide synthesis, bacterial strains and minimal inhibitory con- centration assay}

Peptides were synthesized on a Prelude Peptide Synthesizer (Protein Technologies Inc, AZ) using standard Fmoc solid phase peptide chemistry [1]. The synthesis was performed on Rink Amide AM resin and the amino acid couplings achieved with HCTU (2-(6-Chloro-1H-benzotriazole-1-yl)-1,1,3,3-tetramethylaminium hexafluorophosphate) and NMM (N-methylmorpholine). The peptides were cleaved from the resin using a mixture of $95 \%$ trifluoroacetic acid, $2.5 \%$ triisopropylsilane, $2.5 \%$ water for $3 \mathrm{~h}$ at room temperature and precipitated in cold diethyl ether. After triturating for $2 \mathrm{~min}$, the peptides were collected upon centrifugation and decantation of the ether. The peptides were purified on a Vydac C18 reversed-phase HPLC column $(22 \times 250 \mathrm{~mm}, 5 \mu \mathrm{m})$ over 20 min using a linear gradient of $10-90 \%$ acetonitrile with $0.1 \%$ trifluoroacetic acid at a flow rate of $10 \mathrm{~mL} / \mathrm{min}$ with optical density monitoring at $220 \mathrm{~nm}$. The collected fractions were lyophilised and the identity and purity of the peptides assessed by analytical HPLC and MALDI-TOF mass spectrometry. Peptides were obtained in good yields and with purity greater than $90 \%$.

Escherichia coli K12 MG1655 and Staphylococcus aureus 68 (HER1049) were obtained from the Félix d'Hérelle Reference Center for Bacterial Viruses of Université Laval (http://www.phage.ulaval.ca). Both strains were grown in Trypticase soy broth with agitation at $37^{\circ} \mathrm{C}$. The minimal inhibitory concentration assay was performed as described in [2] and broth microdilution protocol performed in 96-well plates. The bacterial strains were grown overnight at $37^{\circ} \mathrm{C}$ with aeration and diluted to a final concentration of $5 \times 10^{5} \mathrm{cfu} / \mathrm{ml}$ in the assay. The peptides were diluted in sterile water and were tested at the following concentrations: $0,1,2,4,8,16$ and $32 \mu \mathrm{g} / \mathrm{ml}$. The optical density $(600 \mathrm{~nm})$ was followed every 30 minutes for 24 hours with a Synergy 2 plate reader (BioTek Instruments, Inc.).

\section{References}

1. Wellings DA, Atherton E (1997) [4] standard fmoc protocols. Methods in enzymology 289: 44-67.

2. Wiegand I, Hilpert K, Hancock RE (2008) Agar and broth dilution methods to determine the minimal inhibitory concentration (mic) of antimicrobial substances. Nature protocols 3: $163-175$. 